\title{
Hematoma extradural supra e infratentorial
}

\author{
Carlos Umberto Pereira, ${ }^{1,2}$, Marcelo Barreto Barbosa'2, Antonio Carlos Silveira Azevedo², \\ Franklin Roberto Pereira Borges Junior ${ }^{2}$, Allan Valadão de Oliveira Britto ${ }^{3}$
}

Departamento de Medicina da Universidade Federal de Sergipe (UFS) e Hospital de Urgência de Sergipe (HUSE), Aracaju, SE, Brasil.

\section{RESUMO}

Objetivo: Os autores apresentam uma série de casos de hematoma extradural da fossa posterior (HEDFP) com expansão supratentorial. Métodos: O presente trabalho é retrospectivo e descritivo. Foram analisados 14 pacientes com HEDFP de apresentação mista. Resultados: Dos 14 pacientes, 12 são do gênero masculino e dois, do feminino. A média das idades foi de 26,2 anos. Acidente de trânsito foi a principal causa, seguida de queda acidental e agressão física. Escore na escala de coma de Glasgow variou entre 8 e 14. Cefaleia e vômitos foram os principais achados clínicos. Exame de $R X$ simples de crânio demonstrou traço de fratura em $80 \%$ (8/10) dos casos. Tomografia de crânio demonstrou traço de fratura e hematoma extradural mista em todos os pacientes e ressonância magnética em um caso. Cirurgia foi realizada em 12 e tratamento conservador em dois. Dois pacientes foram a óbito. Conclusão: Na presença de fratura no osso occipital, deve-se suspeitar de HEDFP de forma mista. Exames de imagens são importantes no diagnóstico e conduta.

\section{PALAVRAS-CHAVE}

Traumatismos craniocerebrais/terapia, hematoma epidural craniano, hematoma subdural, fossa craniana posterior.

\section{ABSTRACT}

Supra and infratentorial extradural hematoma

Objective: The authors present a case series of HEDFP with supratentorial expansion. Methods: This study is retrospective and descriptive. We analyzed 14 patients with HEDFP presentation mixed. Results: Of 14 patients, 12 males and two females. Mean age was 26.2 years. Traffic accidents were the leading cause, followed by accidental fall and assault. Score on the Glasgow coma scale ranged between 8 and 14. Headache and vomiting were the main clinical findings. Examination showed RX plain skull fracture line in $80 \%(8 / 10)$ of cases. Cranial CT scan showed the fracture line and epidural hematoma mixed in all patients and magnetic resonance one case. Surgery was performed in 12 and conservative in two. Two patients died. Conclusion: In the presence of occipital bone fracture should be suspected HEDFP mixed basis. Imaging techniques are important for diagnosis and management.

\section{KEYWORDS}

Craniocerebral trauma/therapy, hematoma epidural cranial, subdural hematoma, cranial fossa posterior.

1 Professor doutor do Departamento de Medicina da Universidade Federal de Sergipe (UFS), neurocirurgião do Hospital de Urgência de Sergipe (HUSE), Aracaju, SE, Brasil.

2 Neurocirurgião do HUSE, Aracaju, SE, Brasil.

3 Médico radiologista do HUSE, Aracaju, SE, Brasil. 


\section{Introdução}

O hematoma extradural localizado na fossa posterior tem incidência de $4 \%$ a $7 \%$ dos casos de hematoma extradural. ${ }^{1}$ A principal causa é o traumatismo craniano. Encontra-se associado a traço de fratura linear no osso occipital e ocorre por lesão do seio transverso ou sigmóideo. $^{2-6}$

O hematoma extradural da fossa posterior (HEDFP) classifica-se em puro e misto. HEDFP misto são aqueles em que há expansão para a região supratentorial. ${ }^{5,7-9}$ Sua incidência não é conhecida. Tomografia computadorizada (TC) tem sido o exame de eleição, e seu tratamento inicial é cirúrgico e com resultado excelente.

Os autores apresentam sua experiência em 14 casos de HEDFP de apresentação mista e são discutidos causas, achados clínicos e neuroimagens, tratamento e prognóstico.

\section{Pacientes e métodos}

O presente trabalho foi retrospectivo, descritivo e observacional. Foram estudados 14 casos de hematoma extradural da fossa posterior de apresentação mista, no serviço de neurocirurgia do HUSE (Aracaju, Sergipe), durante o período entre janeiro de 2008 e dezembro de 2012. Foram analisados quanto a gênero, idade, causas, apresentação clínica, aspectos tomográficos, tratamento e prognóstico.

\section{Resultados}

Foram estudados 14 pacientes, sendo 12 do gênero masculino e dois do feminino. A média das idades foi de 26,2 anos, variando entre 19 e 44 anos. Acidente de trânsito foi a principal causa (com nove casos), queda acidental (três casos), agressão física (um caso) e desconhecido (um caso). O escore na escala de coma de Glasgow variou entre 8 e 14. Cefaleia e vômitos foram os principais achados clínicos. Exame de RX simples de crânio demonstrou traço de fratura em 80\% (8/10) dos casos. Tomografia de crânio foi realizada em todos os pacientes (Figuras 1 a 5) e ressonância magnética (RM) em um caso (Figura 6). Cirurgia foi realizada em 12 e tratamento conservador em dois. Dois pacientes foram a óbito.

\section{Discussão}

O HEDFP é menos frequente que na região supratentorial. Alguns autores classificam o HEDFP em puro e misto. Puros são aqueles confinados apenas na fossa posterior e mistos quando o hematoma apresenta extensão para região supratentorial..$^{5,7-9} \mathrm{O}$ HEDFP misto é raro. ${ }^{4,6,10}$ Mori et al. ${ }^{11}$ relataram que o HEDFP em adulto pode se estender para a região supratentorial e que na infância tem tendência a ser confinado apenas na fossa posterior. Asanin ${ }^{12}$ relatou que, quando o HEDFP é maior do que $30 \mathrm{ml}$, ele dissemina para o espaço supratentorial. Em nosso trabalho, não foi possível estabelecer sua incidência, tendo em vista que foram selecionados apenas casos isolados de HEDFP misto coletados pelos autores.

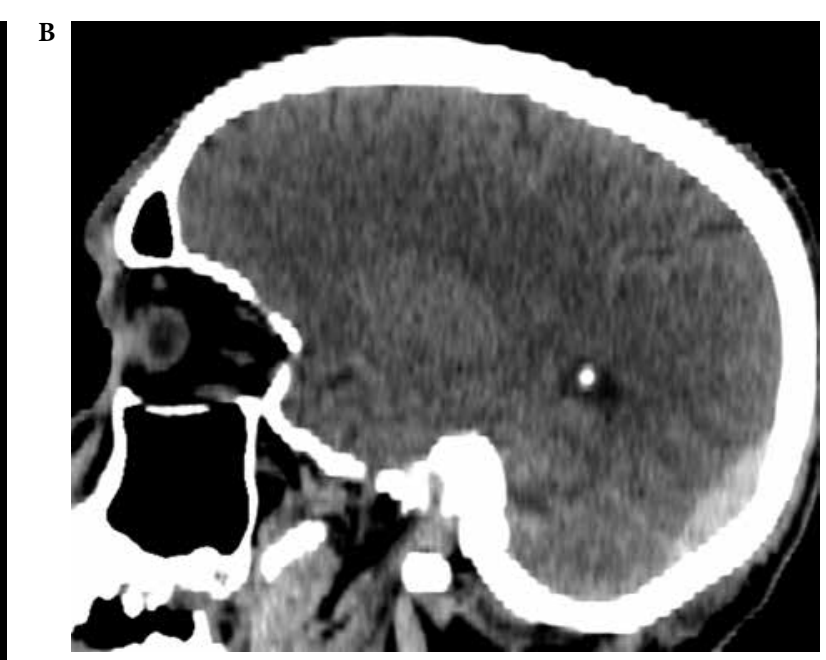

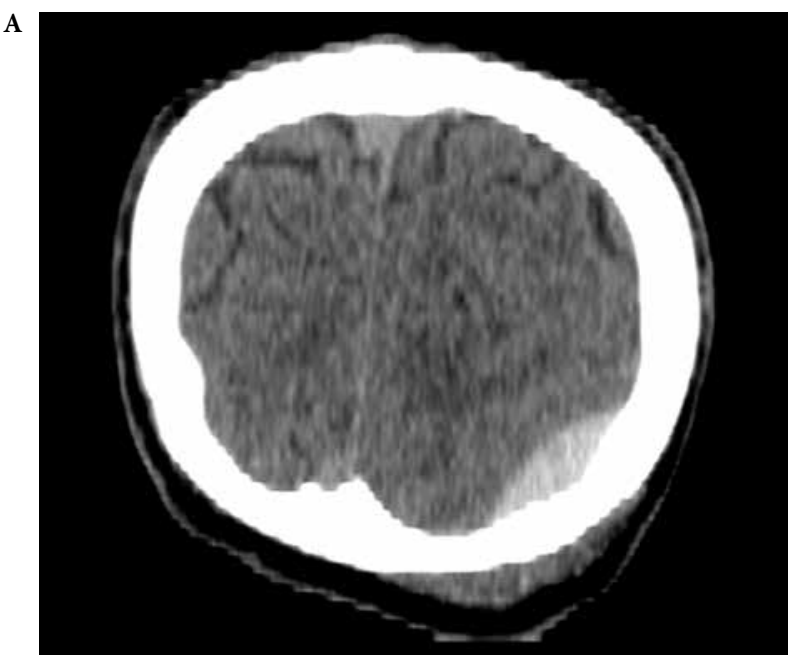

Figura 1 - Tomografia computadorizada do crânio, reformatações nos planos coronal (A) e sagital (B). Coleção laminar biconvexa extraaxial posterior à esquerda, com extensões supra e infratentorial, hiperatenuante e homogênea, compatível com hematoma extradural agudo, de localização mista. 

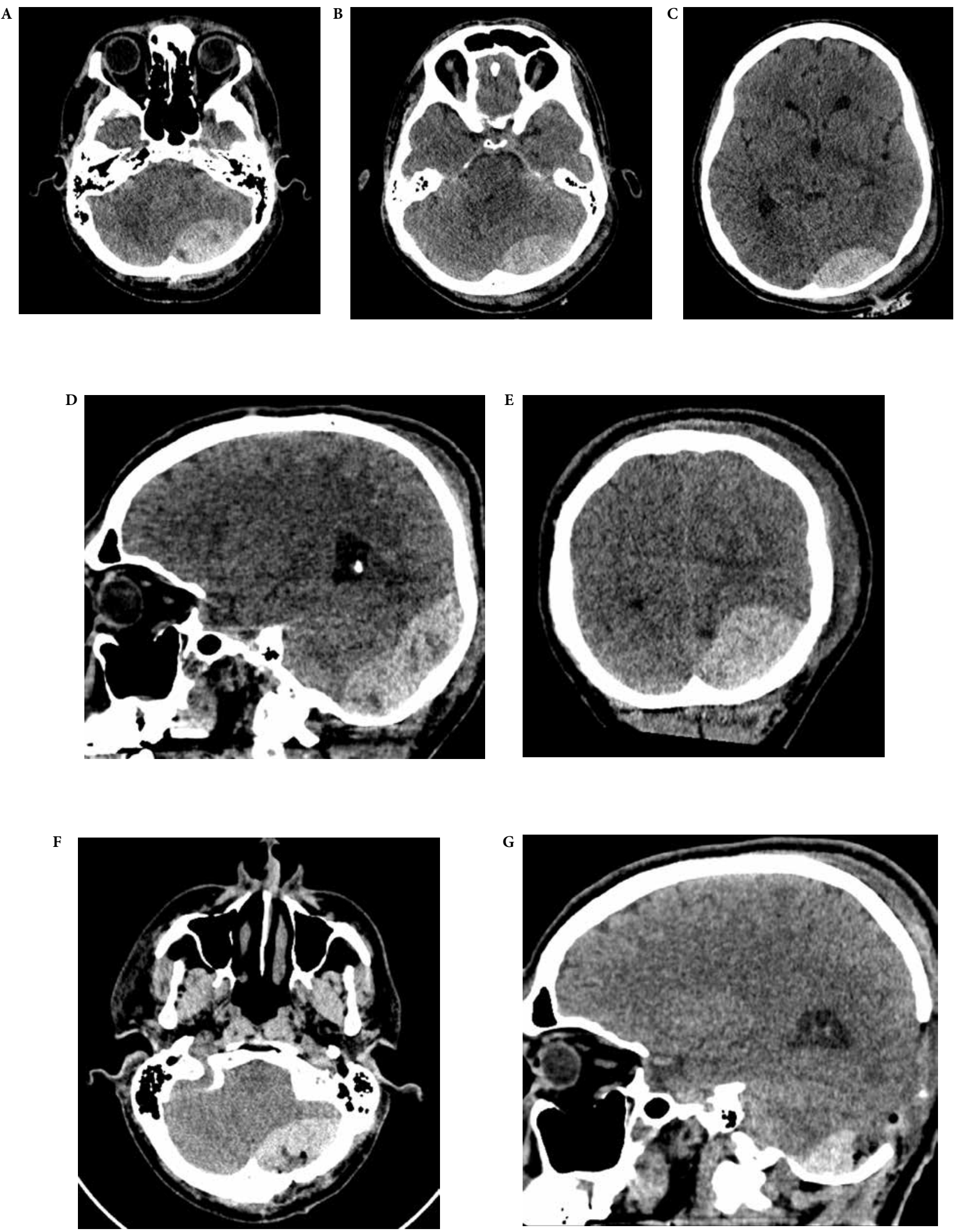

Figura 2 - TC do crânio em cortes no plano transversal (A, B e C), com reformataçães nos planos sagital (D) e coronal (E). Coleção biconvexa extra-axial posterior à esquerda, com extensões supra e infratentorial, hiperatenuante e heterogênea, compatível com hematoma extradural subagudo, de localização mista. Em A e B, observa-se colapso do IV ventrículo e das cisternas na fossa posterior.

TC do crânio pós-operatória em cortes transversal (F) e sagital (G).

Status pós-craniectomia occipital, com hematoma residual na região infratentorial. 


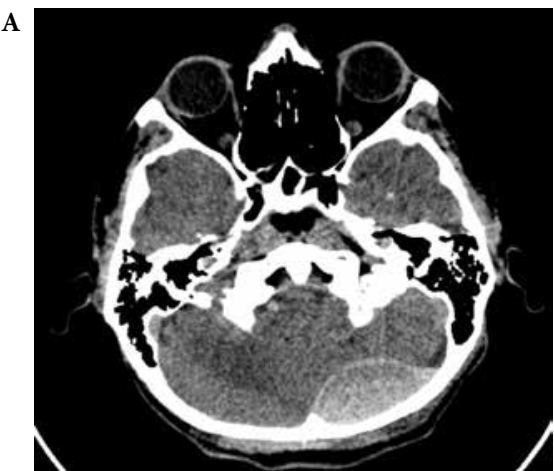

C

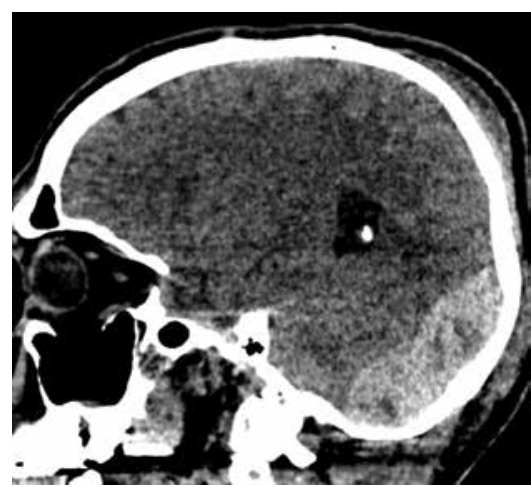

B

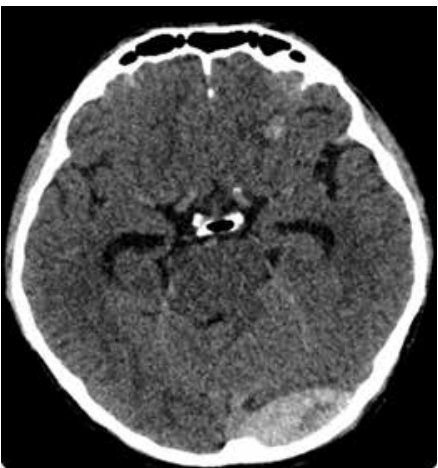

D

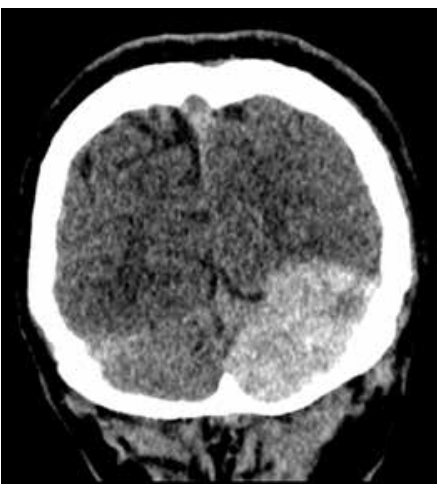

Figura 3 - TC do crânio em cortes no plano transversal (A e B), com reformatações nos planos sagital (C) e coronal (E). Coleção biconvexa extra-axial posterior à esquerda, com extensões supra e infratentorial, hiperatenuante e heterogênea, compatível com hematoma extradural subagudo, de localização mista. Em A e B, observa-se colapso do IV ventrículo e das cisternas na fossa posterior, herniação transtentorial ascendente, além de leve hidrocefalia supratentorial. Em B, nota-se pequeno foco de contusão hemorrágica no lobo frontal esquerdo.
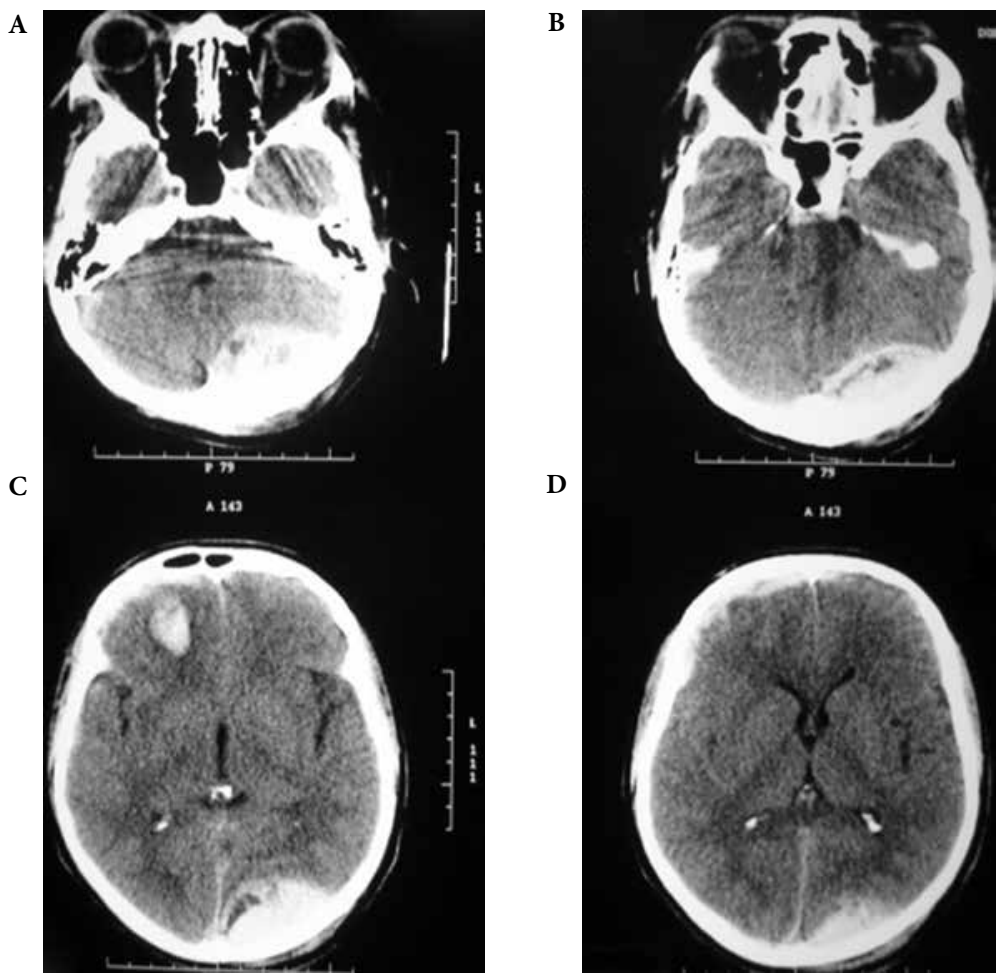

Figura 4 - TC do crânio no plano transversal (A-D). Coleção biconvexa extra-axial posterior à esquerda, com extensões supra e infratentorial, hiperatenuante e heterogênea, compativel com hematoma extradural subagudo, de localização mista. Na figura $C$, observa-se também hematoma intraparenquimatoso no lobo frontal direito. Na figura $D$, nota-se coleção laminar subdural bifrontal. 
A

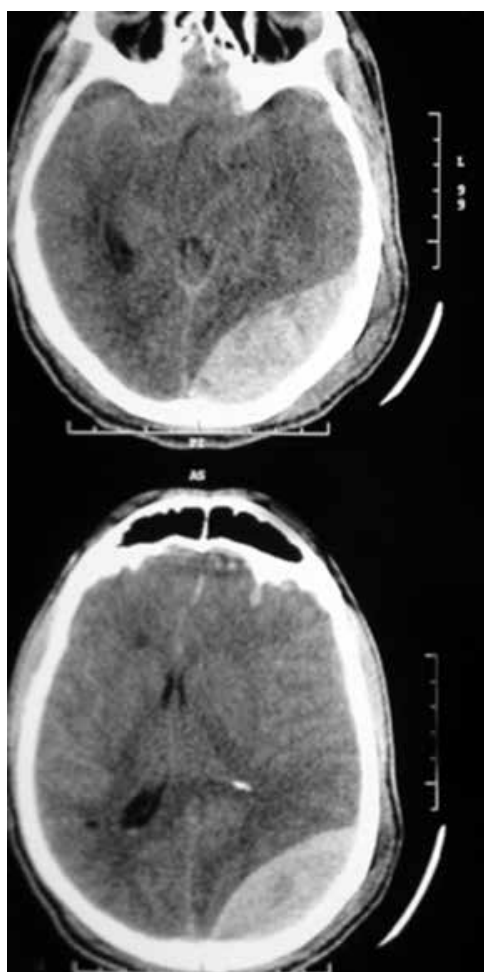

B

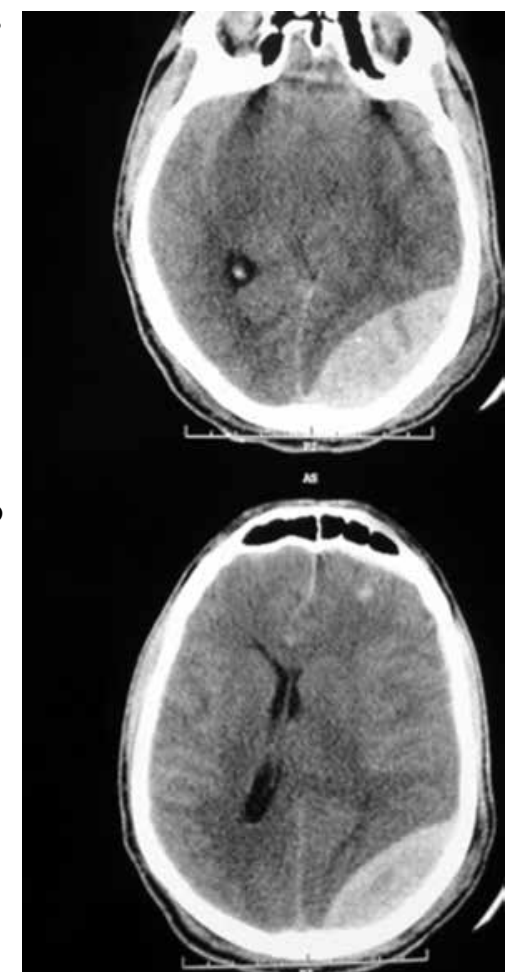

Figura 5 - TC do crânio no plano transversal (A-D). Coleção biconvexa extra-axial posterior à esquerda, com extensões supra e infratentorial, evidenciando o componente supratentorial, hiperatenuante e levemente heterogêneo, compatível com hematoma extradural subagudo, de localização mista. Determina significativas herniações subfalcinas para direita e transtentorial.

A

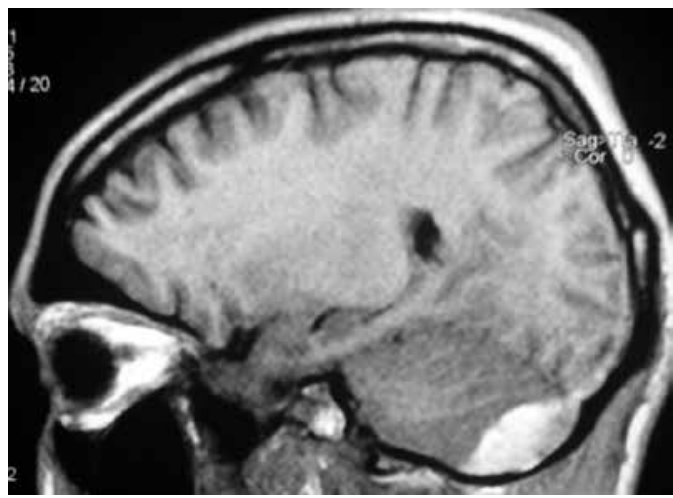

C

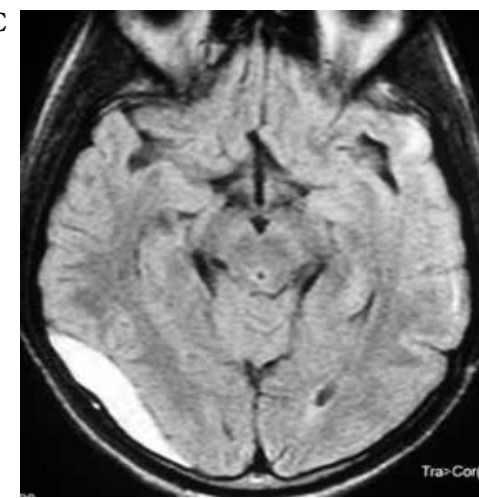

B

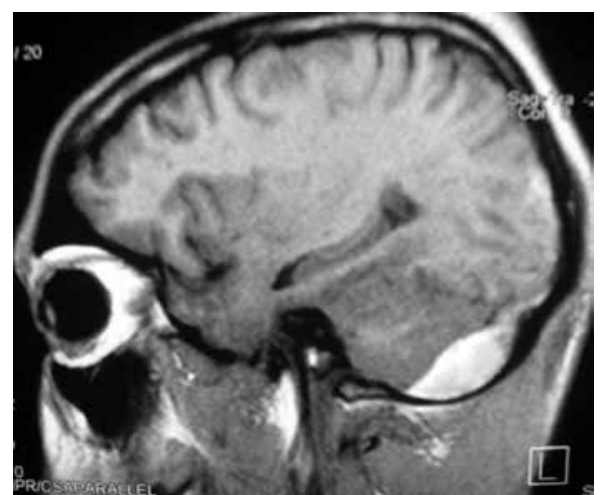

D

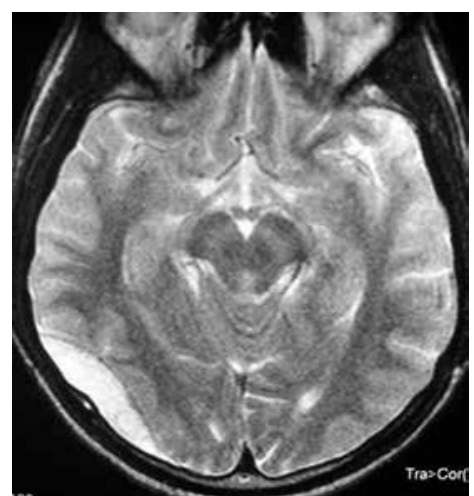

Figura 6 - RM do crânio, planos sagitais ponderados em T1 (A e B) e transversais ponderados em T1 (C) e T2 (D). Coleção laminar biconvexa extra-axial posterior à direita, com extensões supra e infratentorial, apresentando hipersinal em T1 e T2, compatível com hematoma extradural em fase subaguda, de localização mista. 
Geralmente são devidos a acidentes de trânsito e queda acidental, ${ }^{6}$ fato esse observado em nossos pacientes. Resulta em lesões do seio transverso (lateral) ou sigmóideo. ${ }^{10}$

$\mathrm{Na}$ fase aguda, os HEDFP podem ser assintomáticos. ${ }^{6} \mathrm{Na}$ maioria dos casos, são diagnosticados quando existe alto grau de suspeita, com história de trauma na região occipital, fratura do osso occipital e presença de sintomas persistentes e sugestivos de pressão intracraniana elevada, que revela uma lesão expansiva ocupando a fossa posterior. Su et al., ${ }^{13} \mathrm{em}$ sua série de 27 casos de HEDFP, observaram que em oito casos houve deterioração aguda e, desses, três tinham HEDFP misto. Cefaleia e vômitos foram os achados mais comuns em nossos pacientes. A presença de sinais cerebelares e o envolvimento de nervos cranianos têm sido incomuns. Em idosos as manifestações clínicas neurológicas são menos frequentes por causa da atrofia cerebral e também porque o hematoma se desenvolve na região parietal posterior. ${ }^{6,10}$

Em 25\% dos casos de HEDFP misto não existe presença de traço de fratura occipital no exame de raios $\mathrm{X}$ simples de crânio. ${ }^{10}$ Em 10 pacientes deste trabalho que foram submetidos a exame de raios $\mathrm{X}$ simples de crânio, observamos que oito $(80 \%)$ apresentavam traço de fratura na região occipital. O exame de TC tem sido o meio de diagnóstico precoce e de conduta nesses casos. Nossos pacientes foram submetidos a exame de TC, e presença de hidrocefalia ou de dilatação ventricular foi observada em três pacientes, não sendo necessária a realização de shunt. O exame de RM tem sido útil na detecção de lesões no parênquima cerebral ou de trombose de seios venosos durais que pode acompanhar essa lesão. ${ }^{14}$

O tratamento inicial é cirúrgico. Quando realizado exame de TC em casos suspeitos, esses pacientes são tratados de maneira agressiva por meio da drenagem do hematoma e com boa recuperação na maioria dos casos. Otsuka et al. ${ }^{15}$ indicam cirurgia baseados em indicações de achados de TC: máximo de espessura do hematoma maior que $15 \mathrm{~mm}$; cisternas da fossa posterior pobremente visualizadas; marcada deformidade e/ou deslocamento do quarto ventrículo; extensão do hematoma para região supratentorial, resultando em compressão cerebral severa. Contudo, em alguns casos selecionados o tratamento conservador tem sido realizado com vigilância clínica e tomográfica intensa. ${ }^{4,6,16}$ Em nosso trabalho, 12 pacientes foram submetidos à drenagem cirúrgica por meio de craniectomia suboccipital com extensão supratentorial. Presença de descolamento da tórcula foi observada em três casos; segundo vários autores o sinal clássico de descolamento da tórcula é visto na TC em $25 \%$ dos casos. ${ }^{6,17,18}$ Dois pacientes foram submetidos a tratamento conservador por causa do pequeno volume do hematoma, por serem assintomáticos e diagnosticados na fase subaguda. Dois pacientes foram a óbito em decorrência da presença de lesão supratentorial associada e ECG abaixo de 9.

Lui et al. ${ }^{7}$ relacionaram o péssimo prognóstico dos HEDFP quando estão associados a forma mista, obliteração da cisterna perimesencefálica, desvio do quarto ventrículo, escore ECG $<8$ e presença de lesões intracranianas associadas na TC, por exemplo, presença de hidrocefalia, fato esse observado em dois casos deste trabalho.

\section{Conclusão}

Os autores concluíram que traço de fratura cruzando o seio lateral leva à suspeita da presença de HEDFP da forma mista, e um exame de TC deve ser realizado o mais rápido possível para sua identificação e tratamento adequado.

\section{Conflito de interesses}

Os autores declaram inexistência de conflito de interesses na realização deste trabalho.

\section{Referências}

1. Dirim BV, Orük C, Erdogan N, Gelal F, Uluç E. Traumatic posterior fossa hematomas. Diagn Interv Radiol. 2005:11(1):14-8.

2. Garza-Mercado R. Extradural hematoma of the posterior cranial fossa. Report of seven cases with survival. J Neurosurg. 1983;59(4):664-72.

3. Inohue H, Nakagawa $\mathrm{Y}$, Ikemura $\mathrm{M}$, Shinone $\mathrm{K}$, Okada K, Nata M. A subacute epidural hematoma extending over the occipital region and posterior cranial fossa due to a laceration in the transverse sinus. Int $\mathrm{J}$ Legal Med. 2012;126(3):467-71.

4. Is M, Can A, Argül MH. Chronic supra-and infratentorial epidural hematoma. Case report. Turkish Neurosurg. 2006;16:212-3.

5. Prat R, Galeano I. Posterior venous epidural hematoma. Based on 2 cases. Neurologia. 2003;18(1):38-41.

6. Solmaz I, Civelek E, Dinç C, Seçer HI, Temiz Ç, Gönül E. Supra- and infratentorial acute epidural hematoma: a case report. Sinir Sistemi Cerrahisi Derg. 2009;2(3):161-3.

7. Lui TN, Lee ST, Chang CN, Cheng WC. Epidural hematomas in the posterior cranial fossa. J Trauma. 1993;34(2):211-5.

8. Pozzati E, Tognetti F, Cavallo M, Acciarro N. Extradural hematomas of the posterior cranial fossa. Observations on a series of 32 consecutive cases treated after the introduction of computed tomography scanning. Surg Neurol. 1989;32(4):300-3. 
9. Roka YB, Kumar P, Bista P, Sharma GR, Adhikari P. Traumatic posterior fossa extradural haematoma. JNMA J Nepal Med Assoc. 2008;47(172):174-8.

10. Updhyay P, Srivastar A. Supra- and infratentorial acute extradural hematoma. IJNT. 2007;4(1):59-61.

11. Mori K, Handa M, Munemitsu H, Oda Y, Hashimoto N, Kojima M. Epidural hematomas of the posterior fossa in children. Child's Brain. 1983;10(2):130-40.

12. Asanin B. Traumatic epidural hematomas in posterior cranial fossa. Acta Clin Croat. 2009;48(1):27-30.

13. Su TM, Lee TH, Lee TC, Cheng CH, Lu CH. Acute clinical deterioration of posterior fossa epidural hematoma: clinical features, risk factors and outcome. Chang Gung Med J. 2012;35(3):271-80.

14. Osborn AG. Cranoocerebral trauma. In: Osborn AG. Diagnostic neuroradiology. St Louis: Mosby; 1994. p. 204-5.

15. Otsuka S, Nakatsu S, Matsumoto S, Sato S, Motozaki T, Ban S, et al. Study on cases with posterior fossa epidural hematoma - Clinical features and indications for operation. Neurol Med Chir (Tokyo). 1990;30(1):24-8.

16. Gupta PK, Mahapatra AK, Lad SD. Posterior fossa extradural hematomas. Indian J Pediatr. 2002;69(6):489-94.

17. Besson G, Leguyader J, Bagot d'Arc M, Garré H. Extradural hematoma of the posterior fossa. (author's transl). Neurochirurgie. 1978;24(1):53-63.

18. Lê Guyader J, Besson G, Blain F, Bellet M. Angiographic signs of extradural hematomas of the posterior fossa. (author's transl). Sem Hop. 1980;56(17-18):837-41.

Endereço para correspondência

Carlos Umberto Pereira

Av. Augusto Maynard, 245/404,

Bairro São José

49015-380 - Aracaju, SE, Brasil

E-mail: umberto@infonet.com.br 